\title{
Level Crossing Rate and Average Fade Duration for the Beaulieu-Xie Fading Model
}

\author{
Adebola Olutayo, Student Member, IEEE, Hui Ma, Julian Cheng, Senior Member, IEEE, \\ and Jonathan F. Holzman, Member, IEEE
}

\begin{abstract}
Level crossing rate (LCR) and average fade duration (AFD) of a new (Beaulieu-Xie) fading model are analyzed. The characteristic function method is used to derive the LCR for a diversity scheme using maximal ratio combining. The LCR and AFD of the Beaulieu-Xie fading model show improvement beyond the performance levels of the Ricean and Nakagami- $m$ fading models.
\end{abstract}

\section{INTRODUCTION}

$\mathbf{T}$ HE Ricean fading model has gained acceptance due to its ability to characterize wireless systems with a dominant line-of-sight (LOS) channel and multiple non-lineof-sight (NLOS) channels. However, the Ricean fading model has limited flexibility in characterizing the broad range of signal fades from reflections and shadowing. In contrast, the Nakagami- $m$ distribution has been found to be an effective fading model because it includes a flexible fading parameter, allowing it to be adapted for varying levels of severity in signal fades. However, attempts to apply the Nakagami- $m$ fading model to wireless systems with both LOS and NLOS channels have been unsuccessful. This is because the Nakagami- $m$ fading model is a normalized form of the chi-distribution, which cannot define a LOS channel [1]-[3].

Given the benefits and challenges of the Ricean and Nakagami- $m$ fading models, these models were recently merged into a new fading model by Beaulieu and Xie [4]. The Beaulieu-Xie fading model acquires the benefits of the Nakagami- $m$ fading model, in that it has a flexible fading parameter, and the benefits of the Ricean fading model, in that its use of a non-central chi-distribution allows it to characterize both LOS and NLOS channels.

In this work, the level crossing rate (LCR) and average fade duration (AFD) [5] are computed to evaluate the dynamic performance of the Beaulieu-Xie model. The LCR and AFD are key second-order statistics that define the quality of the received signal. The AFD is especially critical in understanding the statistics of error bursts [6]. The characteristic function (CF) method is used to analyze diversity reception via maximal ratio combining (MRC) [7]. Such a method overcomes the analytical complexities of derivations employing the joint probability density function (pdf). We conclude that the LCR and AFD obtained for this model are improved beyond the performance levels of the Ricean and Nakagami- $m$ fading models. To the authors' knowledge, this is the first work that evaluates the dynamic performance of this new fading model with diversity combining. A simple formula is ultimately

The authors are with the School of Engineering, The University of British Columbia, Kelowna, BC, Canada V1V 1V7 (e-mail: julian.cheng@ubc.ca). This research is supported by Natural Sciences Engineering Research Council of Canada (NSERC). presented to show the effect of the LOS component(s), in terms of the signal mean and fading parameter.

\section{Probability Density Function of the Beaulieu-Xie Fading Model}

For the Beaulieu-Xie fading model, it is assumed that we have a non-central chi-distributed random variable (RV), $Y=$ $\sqrt{\sum_{i=1}^{k}\left(\frac{X_{i}}{\sigma_{i}}\right)^{2}}$, where $X_{i}$ is a non-zero Gaussian distributed $\mathrm{RV}$ and $X_{i} \sim \mathcal{N}\left(\mu_{i}, \sigma_{i}^{2}\right), i=1,2, \cdots, k$; with mean $\mu_{i}$ and variance $\sigma_{i}^{2}$, and the $k$ parameter denotes the degree of freedom. The pdf of $Y$ can be obtained by

$$
f_{Y}(y ; k, \lambda)=\frac{\exp \left(-\frac{y^{2}+\lambda^{2}}{2}\right) y^{k} \lambda}{(\lambda y)^{\frac{k}{2}}} I_{\frac{k}{2}-1}(\lambda y)
$$

where $I_{n}(\cdot)$ is the $n$th order modified Bessel function of the first kind and $\lambda$ is the non-centrality parameter, which is related to the mean and variance values of the Gaussian RVs by $\lambda=\sqrt{\sum_{i=1}^{k}\left(\frac{\mu_{i}}{\sigma_{i}}\right)^{2}}$. A new RV can be obtained by normalizing $Y$ by $\sqrt{\frac{2 m}{\Omega}}$ with the parameters $m=\frac{k}{2}$ and $\Omega$. The Beaulieu$\mathrm{Xie}$ fading pdf is given by (2) at the top of the next page [4]. Using the in-phase and quadrature approach [8], one can derive the phase distribution of the Beaulieu-Xie fading model. The parameter $m$ quantifies the severity of the fading, i.e., the higher the value of $m$, the less severe the fading conditions. Ultimately, $m$ controls the shape, $\Omega$ controls the spread, and $\lambda$ impacts the location and height of the mode of the pdf [4]. The performance of this new fading model in different fading conditions is illustrated with symbol error rate curves in [4].

\section{LeVel CRossing RATE}

The LCR and AFD are key metrics to dynamic performance in mobile communication systems [5]. The rate of change of the received signal in mobile communication can be easily related to the crossing (signal) level ${ }^{1}$ and velocity [9]. The LCR, $N_{R}(r)$, is the average number of times a fading signal crosses a given signal level, $r$, within a certain period of time, $t$. Here, the envelope of the received signal, $R(t)$, is subjected to Beaulieu-Xie fading with the pdf in (2). The LCR in the fading environment can be expressed as

$$
N_{R}(r)=\int_{0}^{\infty} \dot{r} f_{R, \dot{R}}(r, \dot{r}) d \dot{r}=\int_{0}^{\infty} \dot{r} f_{R}(r) f_{\dot{R}}(\dot{r}) d \dot{r} \quad r \geq 0
$$

\footnotetext{
${ }^{1}$ Crossing level and signal level are used interchangeably in this work.
} 


$$
f_{R}(r ; m, \lambda, \Omega)=\frac{\exp \left(-\frac{m}{\Omega}\left(r^{2}+\lambda^{2}\right)\right) r^{m}\left(\frac{2 m}{\Omega}\right)}{\lambda^{m-1}} I_{m-1}\left(\frac{2 m}{\Omega} \lambda r\right)
$$

where $f_{R, \dot{R}}(r, \dot{r})$ is the joint pdf of $R(t)$ and its time derivative, $\dot{R}(t)$, and the second equality follows when $R(t)$ is assumed to be independent of $\dot{R}(t)$. Hereafter, for simplicity we will omit $t$. For single branch reception, the pdf of the time derivative of the received signal envelope, $\dot{R}(t)$, is $[10$, eq. (8)]

$$
f_{\dot{R}}(\dot{r})=\sqrt{\frac{1}{2 \pi \dot{\sigma}_{\dot{r}}^{2}}} \exp \left\{-\frac{\dot{r}^{2}}{2 \dot{\sigma}_{\dot{r}}^{2}}\right\}
$$

where $\dot{\sigma}_{\dot{r}}^{2}$ is the time derivative of the variance of $\dot{R}(t)$. The variance of $\dot{R}(t)$ for the Ricean fading model is [10]

$$
\dot{\sigma}_{\dot{r}}^{2}=2 \pi^{2} f_{m}^{2} \sigma^{2}
$$

where $2 \sigma^{2}$ is the Ricean power of the NLOS component(s) and $f_{m}$ is the maximum Doppler frequency. The transformation that unifies the Ricean fading model to the Beaulieu-Xie fading model is $s=\sigma \sqrt{\frac{2 m}{\Omega}} \lambda$ [4], where $s^{2}$ is the Ricean LOS power, $\lambda^{2}$ is the Beaulieu-Xie LOS power, and $\Omega$ is the Beaulieu-Xie NLOS power. Then, $\dot{\sigma}_{\dot{r}}^{2}$ for the Beaulieu-Xie fading model is

$$
\dot{\sigma}_{\dot{r}}^{2}=\frac{\pi^{2} f_{m}^{2} \Omega}{m}
$$

We insert (6) into (4), and (2) into (3), and apply mathematical manipulations to give LCR in (7) at the top of next page.

\section{Average Fade Duration}

The AFD, $T_{R}(r)$, is the average time that the received envelope, $R(t)$, remains below a given crossing (signal) level, $r$, after crossing it in the negative direction [10]. The AFD is

$$
T_{R}(r)=\frac{F_{R}(r)}{N_{R}(r)}=\frac{\int_{0}^{r} f_{R}(t) d t}{N_{R}(r)}
$$

where $F_{R}(r)$ is the cumulative distribution function (CDF) of $R(t)$ and the integral in (8) is expressed as [4, eq. (5)]

$$
\begin{aligned}
\int_{0}^{r} f_{R}(t) d t & =\int_{0}^{r} \frac{\exp \left(-\frac{m}{\Omega}\left(t^{2}+\lambda^{2}\right)\right) t^{m}\left(\frac{2 m}{\Omega}\right)}{\lambda^{m-1}} \\
& \times I_{m-1}\left(\frac{2 m}{\Omega} \lambda t\right) d t .
\end{aligned}
$$

\section{Simulation Results}

The Beaulieu-Xie fading model is simulated using MATLAB to show the effects of various parameters and compare the observations to those of the existing Ricean fading model. Figure 1 shows the normalized LCR curves of the BeaulieuXie, Ricean, and Nakagami- $m$ fading models as a function of the crossing level, $r$. It is seen that the normalized LCR of the Beaulieu-Xie fading model approaches that of the Ricean fading model as $m$ is reduced to 1 , corresponding to increased fading severity, at the same LOS and NLOS powers. The LCR values of the Beaulieu-Xie fading model are lower than those of the Ricean and Nakagami- $m$ fading model. The

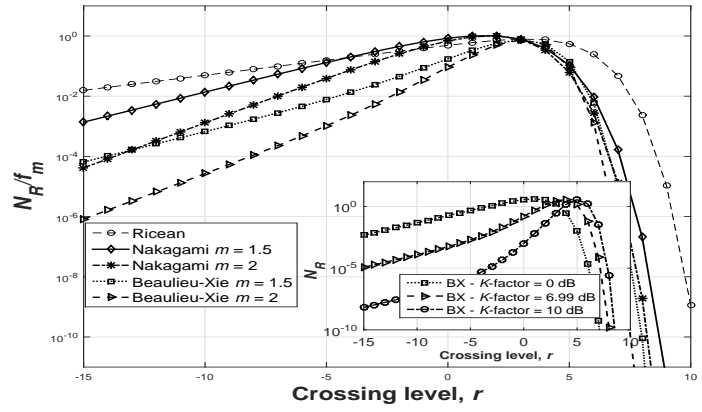

Fig. 1. The normalized LCR curves of the Beaulieu-Xie, Ricean and Nakagami- $m$ fading models versus crossing level, $r$. The Beaulieu-Xie, Ricean and Nakagami- $m$ fading model curves have a total power of $4.88 \mathrm{~dB}$. The Beaulieu-Xie and Nakagami- $m$ curves are shown for fading parameters of $m=1.5$ and 2. Inset: The LCR curves of the Beaulieu-Xie fading model versus crossing level, $r$, with a $K$-factor of 0,5 and $10 \mathrm{~dB}$.



Fig. 2. The LCR curves of the Beaulieu-Xie fading model versus crossing level, $r$, for vehicular speeds of $v=5,30,80,100,140$ and $200 \mathrm{~km} / \mathrm{h}$.

inset figure shows the effect of the $K$-factor on the LCR of Beaulieu-Xie fading. An increase in $K$-factor corresponds to an increase to the LOS power, which leads to lower LCR values. Figures 2 and 3 show the LCR and AFD as a function of the crossing level, $r$, for vehicular speeds up to $100 \mathrm{~km} / \mathrm{h}$. The LCR and AFD are seen to be dependent on the speed of the mobile device, i.e., dependent on the maximum Doppler shift frequency. The LCR increases as $f_{m}$ becomes large while AFD decreases as the $f_{m}$ becomes large. Figure 4 shows the AFD as a function of the crossing level, $r$, for the fading parameter, $m$.

\section{Level Crossing Rate EXPRESSiOn fOR MAXIMAL RATIO COMBINING}

Diversity combining techniques can counter variations in the envelope of a received signal in wireless systems. The LCR and AFD are two important statistics that can be used to analyze such diversity schemes.

In [7], the LCR was calculated using a CF method, as an alternative to the standard pdf method. Using this approach, we define the LCR as the expected number of occurrences per 


$$
N_{R}(r)=\frac{\pi f_{m} \sqrt{\Omega}}{\sqrt{2 \pi m}} \frac{\exp \left(-\frac{m}{\Omega}\left(r^{2}+\lambda^{2}\right)\right) r^{m}\left(\frac{2 m}{\Omega}\right)}{\lambda^{m-1}} I_{m-1}\left(\frac{2 m}{\Omega} \lambda r\right)
$$



Fig. 3. The AFD curves of the Beaulieu-Xie fading model versus crossing level, $r$, for vehicular speeds of $v=0,5,30,80$, and $100 \mathrm{~km} / \mathrm{h}$.

unit time that a stationary random process $R(t)$ crosses the signal level $r$. This is expressed using Rice's formula as [12]

$$
E\left[N_{r}\{R(t)\}\right]=\int_{-\infty}^{\infty}|\dot{r}| f_{R, \dot{R}}(r, \dot{r}) d \dot{r}
$$

where $E[\cdot]$ denotes the expectation operation and $|\cdot|$ denotes the absolute value operator, which is obtained from its joint CF. Note that $|\dot{r}|=\dot{r} \operatorname{sgn}(\dot{r})$, where $\operatorname{sgn}(\cdot)$ is the signum function. The joint $\mathrm{CF}$ of $R(t)$ and $\dot{R}(t)$ is defined as $\Phi_{R, \dot{R}}\left(\omega_{1}, \omega_{2}\right)=E\left[e^{\left(j \omega_{1} R+j \omega_{2} \dot{R}\right)}\right]$, where $j^{2}=-1$. We rewrite (10) using Fourier transforms as (11) at the top of the next page, where $\mathscr{F}[\cdot]$ is the Fourier transform operator, $*$ is the complex conjugate operator, and

$$
\begin{aligned}
& \mathscr{F}[\operatorname{sgn}(\dot{r})]=\frac{2}{j \omega_{2}} \\
& \mathscr{F}\left[f_{R, \dot{R}}(r, \dot{r})\right]=\frac{1}{2 \pi} \int_{-\infty}^{\infty} \Phi_{R, \dot{R}}\left(\omega_{1}, \omega_{2}\right) e^{-j \omega_{1} r} d \omega_{1} .
\end{aligned}
$$

Substituting (12) into (11) gives (13) on the next page [7], [14]. We define the total instantaneous signal-to-noise ratio (SNR) at the output of MRC diversity system as

$$
R=\sum_{l=1}^{L} R_{l}
$$

where $L$ is the number of branches and $R_{l}$ is the instantaneous SNR of the $l$ th branch. Here, $R_{l}$ is expressed as

$$
R_{l}=\sum_{i=1}^{k} \mathcal{X}_{l, i}^{2}
$$

assuming unit noise variance, where $k=2 m$ signifies the degrees of freedom for the diversity system and $m$ is the fading parameter. For the $l$ th branch, $\mathcal{X}_{l, i}$ 's are independent and identically distributed (i.i.d.) Gaussian RVs with equal mean $\mu_{l}$ and equal variance $a_{l}$. Thus, the joint $\mathrm{CF}$ for independent branches can be written as (16) at the top of the next page. For each $l, \dot{\mathcal{X}}_{l, i}$ 's are Gaussian RVs with zero mean and equal variance $b_{l}$. Equation (16) can also be expressed with the following vectors and matrices:

$$
\begin{aligned}
\mathbf{M}_{l} & =\left[\begin{array}{ccccc}
\mu_{l, 1} & 0 & \mu_{l, 2} & \cdots & 0
\end{array}\right]_{1 \times 2 k}^{T} \\
\mathbf{D} & =\left[\begin{array}{cccccc}
\omega_{1} & 0 & 0 & \cdots & 0 & \omega_{2} \\
0 & 0 & 0 & \cdots & \omega_{2} & 0 \\
0 & 0 & \omega_{1} & . & 0 & 0 \\
0 & 0 & \omega_{2} & \ddots & 0 & 0 \\
0 & \omega_{2} & 0 & \cdots & \omega_{1} & 0 \\
\omega_{2} & 0 & 0 & \cdots & 0 & 0
\end{array}\right]_{2 k \times 2 k} \\
\mathbf{C}_{l} & =\left[\begin{array}{cccccc}
a_{l} & 0 & 0 & 0 & 0 & 0 \\
0 & b_{l} & 0 & 0 & 0 & 0 \\
0 & 0 & a_{l} & 0 & 0 & 0 \\
0 & 0 & 0 & \ddots & 0 & 0 \\
0 & 0 & 0 & 0 & a_{l} & 0 \\
0 & 0 & 0 & 0 & 0 & b_{l}
\end{array}\right]_{2 k \times 2 k}
\end{aligned}
$$

where the superscript $T$ denotes the transpose, $\mathbf{M}_{l}$ is a mean vector, and $\mathbf{C}_{l}$ is a covariance matrix. We continue by expressing (16) as [7], [14]

$$
\Phi_{R_{l}, \dot{R}_{l}}\left(\omega_{1}, \omega_{2}\right)=\frac{\exp \left(\frac{-\mathbf{M}_{l}^{T} \mathbf{C}_{l}^{-1}\left[\mathbf{I}-\left(\mathbf{I}-j 2 \mathbf{C}_{l} \mathbf{D}\right)^{-1}\right] \mathbf{M}_{l}}{2}\right)}{\sqrt{\operatorname{det}\left(\mathbf{I}-j 2 \mathbf{C}_{l} \mathbf{D}\right)}}
$$

where $\mathbf{I}$ is a $2 k$-by- $2 k$ identity matrix and $\operatorname{det}(\cdot)$ denotes the determinant. Substituting the vectors and matrices into (17) and applying mathematical manipulations, we obtain

$$
\Phi_{R_{l}, \dot{R}_{l}}\left(\omega_{1}, \omega_{2}\right)=\frac{\exp \left(-4 \mu^{2} \frac{\left(2 b_{l} \omega_{2}^{2}-j \omega_{1}\right)}{\left(1+4 a_{l} b_{l} \omega_{2}^{2}-j 2 a_{l} \omega_{1}\right)}\right)}{\left(1+4 a_{l} b_{l} \omega_{2}^{2}-j 2 a_{l} \omega_{1}\right)^{m}}
$$

for the Beaulieu-Xie fading model. We assume that the instantaneous SNR of each branch is independent and write

$$
\Phi_{R, \dot{R}}\left(\omega_{1}, \omega_{2}\right)=\prod_{l=1}^{L} \Phi_{R_{l}, \dot{R}_{l}}\left(\omega_{1}, \omega_{2}\right) .
$$

The LCR is then obtained by substituting (19) into (13).

Figure 5 shows the LCR as a function of the crossing level, $r$, at the output of a three-branch MRC combiner, using CF methods for Ricean, Nakagami- $m$ and BeaulieuXie fading models. The inset figures show LCR curves for fading parameters of $m=1.5,2,3$, and 4 and identically and independently distributed (i.i.d.) and non-identically and independently distributed (non-i.i.d.) branches. Here, the i.i.d. branches all have Ricean-distributed envelopes and the noni.i.d. have a Beaulieu-Xie distributed envelope in the first branch and Ricean-distributed envelopes in the second and third branches. The average power per branch is $7.64 \mathrm{~dB}$ and the $K$-factor is $10 \mathrm{~dB}$ for the i.i.d. case. The average powers are 7.64, 7.58 and $6.71 \mathrm{~dB}$ for the first, second and third branches in the non-i.i.d., respectively. The Beaulieu-Xie 


$$
E\left[N_{r}\{R(t)\}\right]=\frac{1}{2 \pi} \int_{-\infty}^{\infty} \mathscr{F}[\dot{r} \operatorname{sgn}(\dot{r})]\left\{\mathscr{F}\left[f_{R, \dot{R}}(r, \dot{r})\right]\right\}^{*} d \omega_{2}
$$

$$
E\left[N_{r}\{R(t)\}\right]=-\frac{1}{2 \pi^{2}} \int_{-\infty}^{\infty} \int_{-\infty}^{\infty} \frac{\Phi_{R, \dot{R}}\left(\omega_{1}, \omega_{2}\right)-\Phi_{R}\left(\omega_{1}\right)}{\omega_{2}^{2}} e^{-j \omega_{1} r} d \omega_{1} d \omega_{2}
$$

$$
\Phi_{R_{l}, \dot{R}_{l}}\left(\omega_{1}, \omega_{2}\right)=E\left[\exp \left(j \omega_{1}\left(\mathcal{X}_{l, 1}^{2}+\mathcal{X}_{l, 2}^{2}+\cdots+\mathcal{X}_{l, k}^{2}\right)+j \omega_{2}\left(2 \mathcal{X}_{l, 1} \dot{\mathcal{X}}_{l, 1}+2 \mathcal{X}_{l, 2} \dot{\mathcal{X}}_{l, 2}+\cdots+2 \mathcal{X}_{l, k} \dot{\mathcal{X}}_{l, k}\right)\right)\right]
$$

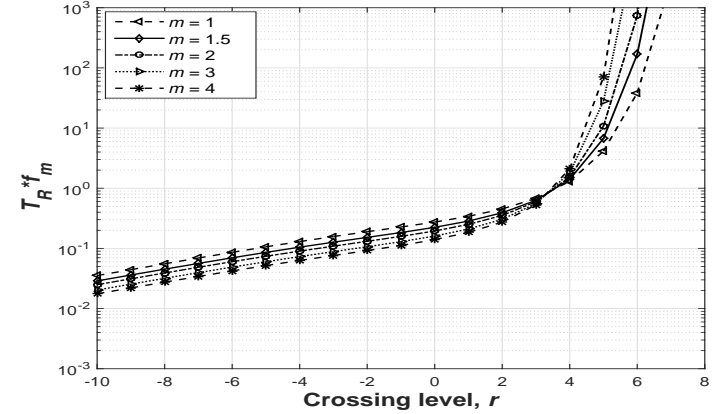

Fig. 4. The AFD curves of the Beaulieu-Xie fading model versus crossing level, $r$, for fading parameters of $m=1,1.5,2,3$, and 4 .

fading model clearly outperforms the Ricean and Nakagami$m$ fading models. Also, it is seen that $m$ impacts the LCR of the system; as $m$ is reduced, the rate of fading in the system increases. A notable difference between the non-i.i.d. and i.i.d. cases is apparent here. This can be a key consideration in system design [7].

\section{CONCLUSIONS}

We studied the LCR for the Beaulieu-Xie fading model and used the CF method to develop an analytical expression for the LCR in an MRC diversity system. The performance of the Beaulieu-Xie fading model was analyzed for various crossing levels, LOS powers, and maximum Doppler frequencies. This fading model can be potentially used to describe high speed train scenario where the LCR becomes relatively insensitive to large Doppler shift. While more evidence needs to be established and show the Beaulieu-Xie model is a better fit to certain realistic communication scenarios, this task further calls for the need to develop efficient parameter estimation of the Beaulieu-Xie fading model.

\section{REFERENCES}

[1] M. D. Yacoub, "Nakagami- $m$ phase-envelope joint distribution: A new model," IEEE Trans. Veh. Technol., vol. 59, no. 3, pp. 1552-1557, Mar. 2010.

[2] N. C. Beaulieu and S. A. Saberali, "A generalized diffuse scatter plus line of-sight fading channel model," in Proc. IEEE ICC 2014, Sydney, NSW, Jun. 2014, pp. 5849-5853.

[3] S. Wyne, A. P. Singh, F. Tufvesson, and A. F. Molisch, "A statistical model for indoor office wireless sensor channels," IEEE Trans. Wireless Commun., vol. 8, no. 8, pp. 4154-4164, Aug. 2009.

[4] N. C. Beaulieu and J. Xie, "A novel fading model in channel with multiple dominant specular components," IEEE Wireless Commun. Lett., vol. 4, no. 1, pp. 54-57, Feb. 2015.

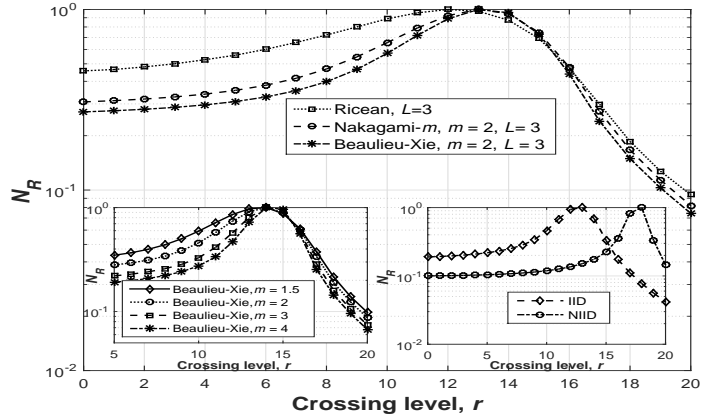

Fig. 5. The LCR curves of the Beaulieu-Xie fading model versus crossing level, $r$, at the output of a 3-branch MRC combiner using the CF method for Ricean, Nakagami- $m$ and Beaulieu-Xie fading models, with an average power of $7.64 \mathrm{~dB}$ per branch. Inset: The LCR curves of Beaulieu-Xie fading versus crossing level, $r$, with fading parameters of $m=1.5,2,3$ and 4 . The LCR curves for i.i.d. and non-i.i.d. branches have a total average power of $12.41 \mathrm{~dB}$ and a $K$-factor of $10 \mathrm{~dB}$ per branch.

[5] D. B. Da Costa, J. C. S. S. Filho, M. D. Yacoub, and G. Fraidenraich, "Crossing rates and fade duration for diversity-combining schemes over $\alpha-\mu$ fading channels," IEEE Commun. Lett., vol. 6, no. 12, pp. 42634267, Dec. 2007.

[6] Z. Cao and Y. D. Yao, "Definition and derivation of level crossing rate and average fade duration in an interference-limited environment, " in Proc. IEEE Vehicle Technology Conf. (VTCFall 01), Atlantic City, NJ, Oct. 2001, pp. 16081611.

[7] A. Abdi and M. Kaveh, "Level of crossing rate in terms of characteristics function: New approach for calculating the fading rate in diversity systems," IEEE Trans. on Commun., vol. 50, no. 9, pp. 1394-1400, Sept. 2002.

[8] M. D. Yacoub, G. Fraidenraich, and J.C.S. Santos Filho, "Nakagami- $m$ phase-envelope joint distribution," Electronic Letters, vol. 41, issue 5, pp. 250-261, Mar. 2005.

[9] T. S. Rappaport, Wireless Communication: Principles and Practise, 2nd Ed. Upper Saddle River, NJ, USA: Prentice Hall, 2001.

[10] X. Dong and N. C. Beaulieu, "Average level of crossing rate and average fade duration of selection diversity," IEEE Commun. Lett., vol. 5, no. 10, pp. 396-398, Oct. 2001.

[11] Z. Hadzi-Velkov, "Level crossing rate and average fade duration of selection diversity with Ricean faded co-channel interferers," IEEE Commun. Lett., vol. 10, no. 9, pp. 649-651, Sept. 2006

[12] A. Abdi and S. Nader-Esfahani, "Expected number of maxima in the envelope of a spherically invariant random process," IEEE Trans. Inf. Theory, vol. 49, no. 5, pp. 1369-1375, May 2003.

[13] S. O. Rice, "Mathematical analysis of random noise," Bell Syst. Tech. J., vol. 23, pp. 282-332, July 1944.

[14] G. L. Turin, "The characteristics function of Hermitian quadratic forms in complex normal variables," Biometrika, vol. 47, pp. 199-201, June 1960.

[15] K. H. Biyari and W. C. Lindsey, "Statistical distributions of Hermitian quadractic forms in complex Guassian variables," IEEE Trans. Inf. Theory, vol. 39, no. 3, pp. 1076-1082, May 1993.

[16] J. G. Proakis and M. Salehi, Digital Communication, 5th Ed. New York, NY, USA: McGraw-Hill, 2007. 